\title{
Real time flow with fast GPU reconstruction for continuous assessment of cardiac output
}

\author{
Grzegorz T Kowalik ${ }^{1 *}$, Jennifer A Steeden ${ }^{1}$, Bejal Pandya ${ }^{1}$, David Atkinson², Andrew M Taylor ${ }^{1}$, Vivek Muthurangu ${ }^{1}$ \\ From 15th Annual SCMR Scientific Sessions \\ Orlando, FL, USA. 2-5 February 2012
}

\section{Background}

Novel real-time phase contrast MR sequences allow assessment of flow during exercise. However, such sequences require a time-consuming reconstruction, which prevents continuous cardiac output monitoring during exercise.

Graphical processing units (GPU) offer the possibility of performing fast reconstruction of real-time MR-data. Such reconstructions would make continuous assessment of cardiac output during exercise possible and uncover new areas in cardiovascular monitoring. The aim of this project is to validate a novel real-time flow sequence with online GPU-based reconstruction.

\section{Methods}

Twenty healthy volunteers underwent aortic flow assessment using spiral SENSE real-time PC-MR (12 interleaves, 4-fold SENSE acquisition, $43 \mathrm{~ms}$ temporal resolution, 13980 frames, acquisition time 10 minutes). Aortic flow was measured continuously during a ramped cycle exercise (2-16W, increased every minute by $2 \mathrm{~W})$.

Real-time data was reconstructed using non-Cartesian iterative SENSE implemented using NVIDIA's CUDA technology on a networked computer equipped with a GTX-480 card. A bidirectional networking scheme was based on CORBA technology (omniORB library).

The absence of data dependencies makes it possible to divide the task into batches of 60 frames with incoming data being buffered on CPU memory. There were 3 reconstruction stages: data transmission onto the external machine, GPU processing and transmission of results to the scanner. The batched-reconstruction allowed for overlapping between these stages and thus

${ }^{1}$ Centre for Cardiovascular MR, UCL, Institute of Cardiovascular Science, London, UK

Full list of author information is available at the end of the article efficient utilisation of the network connection and continuous reconstruction of data.

\section{Results}

The new GPU implementation was tested and compared against our original CPU version. A single batch of 60 flow measurements was retrospectively reconstructed with both versions. GPU implementation showed negligible bias in stroke volume of $-0.4 \mathrm{ml}$ and good limits of agreement -1.9 to $1.2 \mathrm{ml}$. The batch reconstructed 7.7 times faster on the GPU than CPU (15.2 if time required for data transmission is excluded).

Total reconstruction time for the 13980 frames (including transmission and buffering) was $\sim 626 \mathrm{~s}$. Thus, data was available for use $\sim 9 \mathrm{~s}$ after the scan finished (scan duration was $\sim 617 \mathrm{~s}$ ). This is a 556 times speed-up compared to estimated CPU reconstruction (of $\sim 83 \mathrm{~min}$ ).

Fig 1 shows a representative plot of continuous heart rate and cardiac output changes during exercise in one volunteer.

\section{Conclusions}

Continuous assessment of flow during exercise could provide a novel way of assessing hemodynamic responses in patients. Unfortunately, standard real-time MR sequences have long reconstruction times that take over one hour. We have shown that by performing a GPU reconstruction, the data can be clinically available within seconds of the acquisition finishing.

\section{Author details \\ ${ }^{1}$ Centre for Cardiovascular MR, UCL, Institute of Cardiovascular Science, London, UK. ${ }^{2}$ UCL Department of Medical Physics \& Bioengineering, Centre for Medical Image Computing, London, UK.}

Published: 1 February 2012 


\section{Cardiac Output vs Heart Rate}

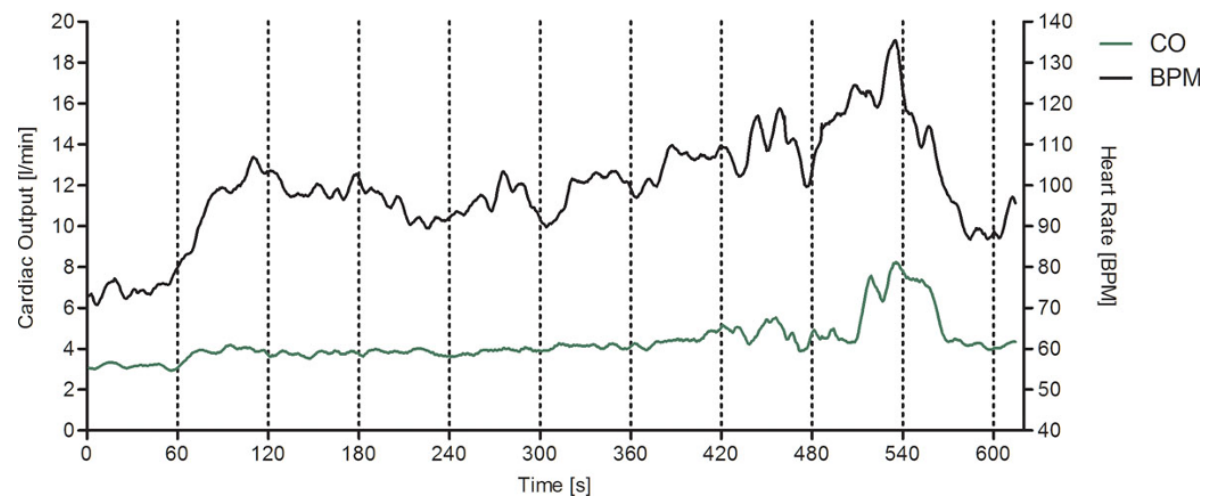

Figure 1

Table 1 Cooperation of iterative SENSE reconstruction implementations. Presented times are per iteration.

\begin{tabular}{cccc}
\hline & CPU [ms] & GPU [ms] & CPU / GPU \\
\hline FFT & 143.83 & 36.97 & 3.9 \\
Gridding & 1337.37 & 29.24 & 46 \\
Preconditioning & 26.47 & 0.5 & 53 \\
Iteration & 3288.32 & 144.83 & 22.71 \\
\hline
\end{tabular}

CPU vs GPU reconstruction.

doi:10.1186/1532-429X-14-S1-W63

Cite this article as: Kowalik et al:: Real time flow with fast GPU

reconstruction for continuous assessment of cardiac output. Journal of Cardiovascular Magnetic Resonance 2012 14(Suppl 1):W63.

Submit your next manuscript to BioMed Central and take full advantage of:

- Convenient online submission

- Thorough peer review

- No space constraints or color figure charges

- Immediate publication on acceptance

- Inclusion in PubMed, CAS, Scopus and Google Scholar

- Research which is freely available for redistribution 